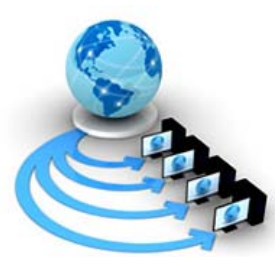

Volume 8, No. 9,November-December 2017

International Journal of Advanced Research in Computer Science

REVIEW ARTICLE

\author{
Available Online at www.ijarcs.info
}

\title{
HANDWRITING COMPARISON IN COURT TRIALS: A NEED FOR FORENSIC UPGRADATION
}

\author{
Urvashi Sharma \\ Asst. Prof. in Comp.Sc. \\ Hans Raj Mahila MahaVidyalaya \\ Jalandhar, Punjab, India
}

\author{
Dr. Vijay Rana \\ Head, DCSE \\ SBBS University \\ Jalandhar, Punjab, India
}

\begin{abstract}
Handwriting comparison is of utmost importance as an evidence in courts as it works both in civil and criminal investigations and enquiries. The law of evidence in India recognizes the role of expert opinion but on the bases of its scientific reliability expert evidence is relied upon by the courts.This paper analyses the judgements of the High Courts and those of the subordinate courts to trace out the importance of handwriting comparison and find out the shortcomings in its process comparing questioned documents with the original/accepted documents. The objective is to identify the scope and areas needing further improvement in its technological or software terms. For this purpose, 10 reported relevant judgements of the High Courts and 36 cases of District courts have been scanned.
\end{abstract}

Keywords: Handwriting Comparison, Signature Verification, Handwriting Forensics, Judgements on Handwriting

\section{INTRODUCTION}

From the High Court appellate judgements, it has been found that in none of such cases, the Court agreed with the opinion of handwriting expert with the result that the concerned accused having allegedly committed crimes like frauds, murder, forgery, abetment to suicide could go scot free because the remaining evidence i.e. excluding of handwritten document was not sufficient to convict i.e. hold guilty to accused, the same being short of the jurisprudentialrequirement of proving the guilt beyond all reasonable doubts. On the other hand, from 36 trial court cases of Jalandhar and Kapurthala District Courts related to testamentary succession (succession by will), transfer of property, partnership agreements, forged certificates and bank dealings, In 7 cases, the courts relied on the opinion of handwriting experts and convicted the accused persons, in 10 more cases the accused were sentenced but not for the reason of handwriting opinion relied upon but the other evidence in the cases being sufficient. However, but in the remaining 19 cases i.e. 53\% the culprits went scot free for non-reliability of opinions given by handwriting experts because the other evidence available in the cases was not sufficient for conviction and/or imposing penalty as per the standard requirements. Taking in totality, in 29 of 36 cases studied, the courts did not rely upon the opinion of handwriting experts, constituting 80.56 percent of the District Court cases studied.

The reason for non-reliability of handwriting comparison as evidence in courts, as studied from the High Court (also referring the High Court reported cases in their support) are science of handwriting comparison is not perfect, possibility of conscious or unconscious bias of such experts in favour of party who engage them, comparison of questioned handwriting with the genuine one is based on experience but not on scientific justifications, the reports not spelling out reasons for arriving at a particular opinion, experts generally being without professional qualifications etc.. When such experts were questioned on the process or procedure followed for arriving at a particular opinion, they disclosed the support of naked eye comparisons or measurements of angular alignments of the writings. The tools of photography, foot rulers, protractors etc. are also used by these handwriting experts. The role of handwriting comparison, as picked up from the court cases, is largely great in court cases because of their relevance in all kinds of cases viz. criminal, civil, revenue, taxation and service matters and law recognizing expert opinion has a relevant piece of evidence.

\section{LAW ON HANDWRITING COMPARISON}

The judgment of a court in any kind of litigation is based on evidence, which can be direct evidence i.e. persons present giving oral evidence or the documents supporting the case or in some cases, it can also be circumstantial evidence where no direct evidence is available. The role of handwriting comparison remains in both categories of situations wherever there is a role ofdocumentary evidence but in cases involving circumstantial evidence, the importance of handwriting comparison gains more prominence and importance. Referring to the Indian Evidence Act, 1872 we find section 60 providing:

"Oral evidence must be direct. Evidence must in all cases be direct that is to say - if it refers to a fact which could be seen it must be the evidence of a witness who says he saw it; if it refers to a fact which could be heard, it must be the evidence of the witness who says he heard it; if it refers to a fact which could be perceived by any other sense or in any other manner, it must be the evidence of a witness who says he perceived it by that sense or in that manner,

If it refers to an opinion or to the grounds on which that opinion is held, it must be the evidence of the person who holds that opinion on those grounds.”.........." (Emphasis given by the researcher) 
Therefore, the handwriting expert giving an opinion will be a witness and may be required to explain grounds for arriving at the opinion. Such grounds may be authenticated by provisions in any treatise on the subject or experimentation scientifically approved. It is basically on this point that science of handwriting is considered lacking in its knowledge or in the qualifications and capabilities of experts. For this reason, perhaps the Supreme Court of India in the famous General Vaidya's assassination case observed: "Science of handwriting is not as perfect as of fingerprints."

The documents are proved by producing the documents alone, however, the execution of such documents will be proved by persons attesting or executing such documents (Ss 68-71, IE Act). If the original document is proved, it is called primary evidence and if it is proved by presenting the certified copy of the original document (if permissible under section 65) it is called proof by secondary evidence.

On the point of proof of signature and handwriting as to the document produced as evidence, section 67 reads as under:

"If a document is allowed to be signed or to have been written wholly or in part by any person, the signature or the handwriting of so much of the document as is allowed to be in that persons handwriting must be proved to be in his handwriting."

To confirm that the document or writing in the document is that of the person who is being claimed to have written the same, the court is competent to obtain handwriting or signatures of such persons and compare the two by itself or through the opinion of a handwriting expert. Section 73 of the Evidence Act provides therefor as:

"73. Comparison of signature, writing or seal with others admitted or proved - In order to ascertain whether a signature, writing or seal is that of the person by whom it purports to have been written or made, any signature, writing, or seal admitted or proved to the satisfaction of the court to have been written or made by that person may be compared with the one which is to be proved although that signature writing or seal has not been produced or proved for any other purpose.The Court may direct any person present in Court to write any words for the purpose of enabling the court to compare the words or figures so written with any words or figures alleged to have been written by such person.This section applies also with necessary modifications, to finger impressions (fingerprints).”

The importance of documents creating contractual liabilities, transfer of property and the like lies in producing such documents which can further be challenged as to whether the same are genuine i.e. actually executed or not i.e. not executed. For the purpose of evidence in court proceedings, section 144 lays down:

"144 Evidence as to matters in writing - Any witness may be asked whilst under examination, whether any contract grant or other disposition of property, as to which he is giving evidence, was not contained in a document, and if he says that it was, or if he is about to make any statement as to the contents of any document, which, in the opinion of the
Court, ought to be produced, the adverse party may object to such evidence being given until such document is produced, or until facts have been proved which entitle the party who called the witness to give secondary evidence of it."

\section{MORE AGREEMENT ON SIGNATURES THAN HANDWRITTEN DOCUMENTS}

Signature is considered as the most common way for humans to acknowledge their identity which has social and legal acceptance. The use of signatures for the verification of a person causes a problem due to their vulnerability nature to reproduction so that the forger can counterfeit his identity. Variations caused by the natural process also pose a problem in comparison. Complexity is another factor due to which the efficiency and the performance of the verification systemare affected. Such complexity also arises from simulation process.

Signature verification is of two types: offline (static) i.e. signatures using pen and paper and online (dynamic) where the signatures are taken by some digitizing system like tablet [1]. Feature extraction, the fundamental task in the offline signature verification, is considered as the most challenging task. One method is by imposing grids on the image. After that the shape, statistical and structural properties of the image are measured. Another wayadopted which is mostly used is by comparing the shape. This selection method takes basic properties of the signature trace into account. The electronic or dynamic signatures have one bigadvantage over paper-pen offline signatures that they can capture the transitory features of the signature which can provide important information todifferentiate between the simulated and authentic signatures. One cannot directly analyse the dynamic features of the pen-paper signatures but can infer it. In case of electronic signatures, features can be analysed by visualizing the dynamics of writing by re-playing the process of signing in real time in order tostudy it in detail [2]. Also the direction of strokes and the sequence can be knownfrom its temporal information. Offline handwritings offer various indicators for this, butto reach a clear conclusion sometimes become impossible.

Offline signature verification techniques can be implemented using two approaches: writer dependent and writer independent [3]. In case of writer dependent, the system is trained with genuine and forged signatures of a particular writer and the system makes a decision based on the similarity or dissimilarity between the questioned signatures and the original signatures of the signer. Its disadvantage is that the system has to be retrained every time the new writer is introduced. In case of writerindependent, the signature verification system is modelled for a generic system that can be tested on any writer [4]. An efficient and effective technique for feature extraction should be able to extract information like curvilinear nature of strokes, pixel connectivity, black pixel density, etc. that can properly describe the signature and thus making the feature set based on neighbouring pixel surroundedness [5].

Over the last decade, researchers have proposed different methods for offline signature verification. Yet, distinguishing between forged signatures and genuine 
signatures still remains a challenging task but the error rates have dropped significantly in the last few years. And this is because of the advancements in Deep Learning applied to it. Several approaches have been used for offline signature verification like Hidden Markov Models (HMM) [6] in which a grid is used to divide the signatures to extract the features form different cells and subsequently quantized in a codebook. Support Vector Machines (SVMs) [1] are most effective classifiers for signature verification for both writerindependent and writer-dependent classifications. But this model attempts to model only single class like of genuine signatures. However, less number of genuine signatures imposes a challenge for this strategy. Neural Networks (NN) are also used to classify between original signatures and forged. In case of NN, multiple networks are trained on feature extraction at different resolutions and another network is used to make a decision based on the outputs of these networks. In Deep Multitask Metric Learning approach (DMML), the system learns to compare two signatures, by learning a distance metric between them. Feedforward NN is used where the bottom layers are shared among all users and the last layer is specific to each individual.

Many software have been developed for the dynamic and static signature verification like iSign, CICs Sign It, BioPen System, SOFTPRO SignDoc, WonderNet'sPenDocs, SIGNificant Online Client, SIGNificant Offline Client, Newgen's ASV, Parascript'sSignatureXpert, Topaz's SigCompare, Softpedia Signature Verification, TIS's ASV, HelloSign and many more whereas the software available for handwriting comparison are still very limited like CedarFox, NeuroScript'sMovAlyzeR\&ScriptAlyzeR and some companies providing these services like eurofins, LGC Group, Q9 Consulting, Inc.

In addition to standard electronic devices for signature acquisition like tablets and scanners, there is a need to introduce new methods like camera based signature acquisition using webcams which is very convenient and inexpensive in a way that user will be convenient to sign using simple pen instead of electronic pen [7, 8]. Also the signature verification on mobile devices is not very efficient because the input area of mobile is very small and it provides poor sampling frequency [9, 10, 11]. In it the person has to use the touch screen for signing instead of natural paper which is not very convenient.

There are two aspects in the problem of modelling of signature: one, its stability and another complexity [3]. The studies have shown that there are some features of the signature and even of handwriting that remainstable with time but there are some other features also that change significantly with time such as the advancement in signer's age. Signature complexity is the scale with which a forger can simulate the signatures with ease or with difficulty. The analysis of each and every characteristic of the handwriting is a researchable area. The future research may also study the changing factors of normal persons as well as of abnormal persons i.e. suffering from disabilities or illness.

The task of handwriting comparison becomes more onerous for many reasons. First, the document written is long that leaves a scope for variations in the letters, their spacing, size, marginalisation, tilt, etc., etc. Where as in signatures one writes same letter in the same shape, size and style but in a document, the same person changes styles, shapes and sizes for the same letter. Moreover, the mood of the person also brings about changes in the handwriting. The change of the situation, posture of the writer, quality of the pen and paper may also affect the handwriting. When change is the law of nature, it reflects more in long writings than it appears in signatures of a person over a time gap.

Forensic efforts have been made for scientific comparison of handwritten documents. The tools like CEDAR FOX, FISH, NeuroScript'sMovAlyzeR\&ScriptAlyzeR have been developed and the processes like Hidden Markov Models (HMM), DTW (Dynamic Time Warping), have been devised for placing handwriting comparison on a scientific platform but for the reasons of inherent difficulties, handwriting comparison has not attained the same scientific approval as is with the signatures, although both are not considered foolproof before the judicial courts. The forensic work on handwriting needs to be done much more vigorously because scientific lag causes much social and economic loss as an evidence in court cases.

The comparison of handwriting is a challenging task because handwriting is a non-rigid shape. Some factors in handwriting evaluation are subjective in nature but it does not mean that the results of comparisons conducted properly will be either faulty or deceptive. The studies [12] have shown that professional forensic document examiners (FDEs)surpassnon-professionals on handwriting verification. The set of features varies from examiner to examiner as one examiner may consider some featuresto be irrelevant and the other examiners may apply different methods for the estimation of their values.

Studies have shown that the automatic tools for handwriting comparison are less successful than using CAD software with examiners [12]. Such failure may be due to the divergence between the measurements estimated by the experts and by the software.

In case of handwriting comparison, the quality of the scanner is also important because the image acquired with common scanner tend to lose information about microscopic details.

\section{CONCLUSION}

The task of developing forensic tools for handwriting and signature comparison is certainly a challenging one but it is not difficult. More scientific efforts in concerted and coordinated terms should be made to develop the tools. Blending of available tools for signature comparison and handwriting verification may be helpful to develop more effective and efficient tools. MATLAB may come to our technological rescue but after development of any tool, its repeated testing as stepping stones of efficiency will certainly bear fruit to our scientific urge and effort.

\section{REFERENCES}

[1] L. Batisa, E. Granger and R. Sabourin, "Dynamic selection of generative-discriminative ensembles for off-line 
signature verification”, Pattern Recognition, Elsevier Science Inc., Vol. 45, Issue 4, pp 1326-1340, April 2012.

[2] J. Heckeroth, C.D. Boywitt, "Examining Authenticity: An Initial Exploration of the Suitability of handwritten Electronic Signatures”, Forensic Science International, Elsevier, pp 144-154, 2017

[3]Impedovo, Donato \& Pirlo, Giuseppe \&Plamondon, Réjean., "Handwritten Signature Verification: New Advancements and Open Issues". Proceedings International Workshop on Frontiers in Handwriting Recognition, IWFHR.367-372. 10.1109/ICFHR.2012.211, 2012.

[4] Y. Guerbai, Y. Chibani and B. Hadjadji, "The effective use of the one-class SVM classifier for handwritten signature verification based on writer-independent parameters", Pattern Recognition, Elsevier Science Inc.,Vol. 48, Issue 1, pp 103-113, January 2015.

[5] R. Kumar, J.D. Sharma, Bhabatosh Chanda, "Writerindependent off-line signature verification using surroundedness feature, Pattern recognition Letters 33, Elsevier, pp 301-308, 2012.

[6] Edson J.R. Justino, A. El. Yacoubi, F. Borotolozzi and R. Sabourin, "An off-line signature verification system using HMM and graphometric features", $4^{\text {th }}$ IAPR International Workshop on Document Analysis Systems (DAS), pp 211-222, 2000
[7] D. Maramatsu, K. Yasuda, T. Matsumoto, "Biometric Person Authentication Method Using Camera-based Online Signature Acquisition,” Proc. 20th ICPR, Istanbul, Turkey, 23-26 Aug. 2010, pp. 46-50.

[8]S. Shirato, D. Maramatsu, T. Matsumoto, "Camera-based online Signature Verification System: Effects of Camera Position”, World Automation Congress, IEEE Xplore, 2010

[9] S. J. Elliott, "Differentiation of Signature Traits vis-à-vis Mobile- and Table-based Digitizers”, ETRI Journal, vol. 26, no. 6, pp. 641-646, December 2004.

[10] F. Zoebisch, C. Vielhauer, "A test tool to support brute-force online and offline signature forgery tests on mobile devices,” Proc. IEEE Int'l Conf. on Multimedia and Expo 2003 (ICME), vol. 3, Baltimore, USA, 2006, pp. 225228.

[11]C. Vivaracho-Pascual, J. Pascual-Gaspar, "On the Use of Mobile Phones and Biometrics for Accessing Restricted Web Services”, IEEE T-SMC - Part C, Applications and Reviews, March 2012, Vol. 42, No. 2, pp. 213 - 222.

[12] A. Parziale, A. Santoro, A. Marcelli, A.P. Rizzo, C. Molinari, "An Interactive Tool for Forensic Handwriting Examination”, IEEE Int'l Conf. on Frontiers in Handwriting recognition (ICFHR), pp 440-444, 2014 